\title{
Beyond Dome C
}

\author{
J.W.V. Storey, M.C.B. Ashley, M.G. Burton, and J.S. Lawrence
}

School of Physics, University of New South Wales, NSW 2052, Australia

\begin{abstract}
A well-focused research program over the past decade has shown that the South Pole has many remarkable characteristics that are particularly favorable for astronomy. These include the very cold, dry atmosphere and the vanishingly small free-air turbulence. Dome $\mathrm{C}$, site of the new French/Italian station Concordia, has all of these attributes plus the added advantage of very low ground-level wind speeds. Higher on the plateau, locations such as the 4200 $\mathrm{m}$ high Dome A may well represent the ultimate ground based astronomical observing sites.
\end{abstract}

The exceptional conditions that make the Antarctic plateau so attractive to astronomers are well known. It is very cold (temperatures can drop below $-80^{\circ} \mathrm{C}$ ) and reasonably high (Dome $\mathrm{A}$ is at $4200 \mathrm{~m}$ ), leading to extremely low levels of precipitable water vapor and thermal emission from the sky.

What is less well know is that the ground-level wind speeds are also very low $\left(2.8 \mathrm{~m} . \mathrm{s}^{-1}\right.$ average at Dome C) and that these winds remain low throughout the entire atmospheric column. This results in a very low turbulence, and that remaining turbulence is confined to a low (100 m scale) boundary layer.

The South Pole is now firmly established as one of the world's premier observing sites (Storey et al. 2002). Over the next few years, Concordia station is set to take its place as an equally attractive draw card for astronomers, as major facilities are constructed to complement those at South Pole.

However, it is natural to ask: what lies beyond? Almost $1 \mathrm{~km}$ higher than Dome C, Dome A can be expected to have essentially zero wind for much of the time and to have precipitable water vapor levels that can drop below 50 microns. These conditions would make possible a wide variety of experiments that are currently only possible from balloons, high-altitude aircraft, or space.

At the present time there is no infrastructure at Dome A, nor any welldefined plans to establish any. It is likely that any observatory to be placed there would be a robotic facility, serviced annually from the South Pole or a coastal station such as Davis. Adaptive-optics aside, modern astronomical instruments are vastly more complicated than the telescopes they are attached to. In a benign environment, such as exists at Dome A, it is reasonable to expect that a robotic telescope would be no more demanding to operate than any existing large-scale instrument at a conventional site.

With little turbulence and very low wind speeds, requirements for adaptive optics are dramatically simplified. It is likely that even large optical/IR telescopes would need only a simple tip-tilt or low order AO system, using just a single natural guide star.

The first step in the exploration of Dome A is to go there. It is remarkable that what is potentially the best astronomical site on earth has never been visited 
(although a Chinese traverse team have come close). An initial expedition should install an Automated Weather Station, plus additional micro-power instruments to measure cloud cover and boundary-layer turbulence.

The next step would be the installation of a comprehensive site-testing facility similar to the AASTINO (Lawrence et al. 2003). Running autonomously for a full year and communicating with the world via Iridium satellite, an AASTINO at Dome $\mathrm{A}$ would not only fully characterize the site but would also represent a prototype of the type of autonomous astronomical observatory that might ultimately be installed there.

A preliminary instrument suite to deploy to Dome A might include:

- an acoustic radar, to measure boundary-layer turbulence,

- a MASS (Multi-Aperture Scintillation Sensor), to measure the distribution of turbulence throughout the atmosphere,

- a mid-infrared spectrometer, to measure sky brightness and opacity from 3-30 micron,

- a sub-millimeter Fourier Transform Spectrometer to measure sky brightness and opacity,

- a near-infrared photometer, to measure sky brightness in the 2.35 micron "cosmological window".

Plans are already underway to include an exploration of the astronomical potential of Dome A as part of the International Polar Year of 2007. Regardless of what is discovered there, the well-established advantages of South Pole and Concordia Station will inevitably lead to a continued rapid expansion in the astronomical activities at those existing sites.

Ultimately, however, Dome A may prove to be simply irresistible.

\section{References}

Lawrence J.S., Ashley, M.C.B., Burton, M.G., Calisse, P.G., Dempsey, J.T., Everett, J.R., Maher, O., Storey, J.W.V., and Travouillon, T. 2003, The AASTINO: Automated Astrophysical Site Testing Invincible Observatory, Memorie della Societ Astronomica Italiana, 2, 217

Storey, J.W.V., Ashley, M.C.B., and Burton, M.G., 2002, Antarctic site testing, in Astronomical site testing in the visible and radio range, ASP Conf. Series, Eds.

Z. Benkhaldoun, Z., Munoz-Tunon, C. and Vernin, J., 266, 524 